\title{
Pain relief with intracameral mepivacaine during phacoemulsification
}

François A Malecaze, Sylvie F Deneuville, Bernard J Julia, Jean G Daurin, Eric M Chapotot, Hélène M C Grandjean, Jean L Arné, Olivier Rascol

\begin{abstract}
Aim-To assess the efficacy and safety of an intraoperative intracameral injection of mepivacaine, administered when patients experienced pain during the course of cataract surgery under topical anaesthesia.

Methods-This is a prospective placebo controlled double masked randomised clinical trial. 50 eyes were included; 25 receiving the active compound and 25 receiving placebo. Mepivacaine $(2 \%, 0.4$ $\mathrm{ml}$ ) or placebo was administered intraoperatively under the iris of the patients who experienced pain during the course of phacoemulsification in spite of previous topical anaesthesia. Efficacy was evaluated by the patients themselves using a five point subjective pain rating scale, the Keele verbal pain chart. Safety was measured by assessing intraocular inflammation (clinical evaluation and laser flare meter), intraocular pressure, and endothelial cell count.
\end{abstract}

Results-The pain rating score significantly diminished after intracameral injection in the mepivacaine group (mean

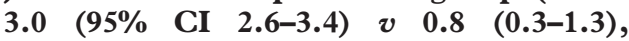
$\left.\mathrm{p}<10^{-4}\right)$ ) while remaining unchanged in the placebo group $(2.9(2.6-3.2) \quad v 2.9(2.5-$ $3.3)$ ), the mean reduction in pain score being significantly different between the two groups $\left(p<10^{-4}\right)$. There was no indication of increased postoperative ocular inflammation, intraocular pressure change, or endothelial cell loss.

Conclusions-These results suggest that it may not be necessary to systematically add intracameral anaesthesia with topical anaesthesia for cataract surgery. An intraoperative intracameral injection, performed only in patients who happen to suffer during surgery, is safe and effective. (Br F Ophthalmol 2000;84:171-174)

Toulouse, France

H M C Grandjean

Department of Clinical

Pharmacology and

Clinical Investigation

Centre, University

Hospital, Toulouse,

France

O Rascol

Correspondence to: Dr F Malecaze, Department

of Ophthalmology,

University Hospital of

Purpan, Place Dr Baylac,

31059 Toulouse, France

Accepted for publication 6 September 1999 ye surgery. Obtaining analgesia by controlle preoperative sedation presents, in addition to the risk of paradoxal agitation, the disadvantage of decreasing patient cooperation, which is important in this type of surgery.

Further systematic anaesthesia by an intracameral injection of lignocaine (lidocaine) has been recently recommended to complete topical anaesthesia. ${ }^{4-7}$ However, since less than $10 \%$ of the patients undergoing cataract surgery under topical anaesthesia usually suffer pain, we wondered whether it was necessary to perform an intracameral injection systematically in every patient. An alternative procedure could be to give such complementary anaesthesia only in those patients who experience pain during surgery.

Therefore we decided to perform a prospective placebo controlled double masked study to evaluate this procedure.

\section{Patients and methods}

\section{OBJECTIVE}

This prospective randomised double masked study was designed to compare with placebo (a balanced salt solution, BSS) the efficacy and safety of an intracameral injection of unpreserved mepivacaine, a locally effective anaesthetic drug with a long duration of action when administered intraoperatively, ${ }^{8-10}$ as soon as patients under topical anaesthesia experienced substantial pain during phacoemulsification.

\section{PATIENT SELECTION}

This study was carried out on patients referred to the department of ophthalmology of Toulouse University Hospital for cataract surgery. All patients were operated in the same manner under similar topical anaesthesia by the same surgeon (FM). All patients operated by this surgeon were allowed the possibility of participating in the trial. Patients were included if they gave informed signed consent and if they had substantial pain during surgery (see "Protocol" below). Patients were excluded from entering the study if they did not give informed consent or if they had a history of ocular disease such as uveitis, glaucoma, corneal disease, or diabetic retinopathy. They were also excluded if their preoperative endothelial cell count was below 1600 cells $/ \mathrm{mm}^{2}$, or if they presented a striking non-age related pleomorphism or polymorphism, because we did not wish to administer a new drug in an affected eye. Patients were excluded if there were any intraoperative surgical complications before the injection of the drug. 
ASSESSMENT CRITERIA

Efficacy criteria

The main assessment criterion for efficacy was the difference in a subjective pain score assessed verbally by the patient during phacoemulsification, before and after the intracameral drug injection. After previous instruction, patients were asked to rate their experience of pain on the Keele verbal pain chart. ${ }^{11}$ This scale allowed them to score pain in a semiquantitative way: $0=$ no pain, $1=$ minimal pain, $2=$ moderate pain, $3=$ severe pain, $4=$ agonising pain.

A second efficacy criterion was the proportion of patients requiring complementary local anaesthesia or general sedation, in spite of the intracameral injection.

\section{Safety criteria}

We assessed four safety criteria.

Endothelial cell loss: Central corneal endothelial cells were counted preoperatively, and 3 months after surgery, in each patient using a specular microscope (Topcon 2000). Endothelial cell loss was expressed as a percentage of the preoperative cell density.

Postoperative inflammation: The amount of postoperative inflammation was assessed by a laser flare meter (Kowa FM 500) preoperatively and on days 1,7 , and 20 after surgery. The degree of postoperative inflammation was also clinically assessed by individually grading the aqueous cellular reaction and aqueous flare, according to the criteria of Hogan et al. ${ }^{12}$ Intraocular pressure: Intraocular pressure (IOP) measurement was recorded using a HaagStreit Goldmann applanation tonometer preoperatively and on days 1,7 , and 20 after surgery.

Haemodynamic variables: During surgery heart rate, systolic and diastolic blood pressure, and haemoglobin oxygen saturation were monitored automatically (Critikon, Ohmeda oximeter).

PROTOCOL

All the patients received, 2 hours before surgery, oral premedication by midazolam, 3-6 $\mathrm{mg}$ according to age, to allow the patient comfort and sufficient alertness. The topical anaesthesia consisted of $2 \%$ bupivacaine, one drop every 5 minutes half an hour before surgery. Phacoemulsification surgery was performed through a self sealing $3.2 \mathrm{~mm}$ clear corneal incision with an implantation of a foldable silicone intraocular lens (IOL). As soon as the patient complained of pain the surgeon asked him to rate his pain using the Keele verbal pain chart. If the pain was rated 2 or more on this scale, $0.4 \mathrm{ml}$ of the assigned drug (placebo or mepivacaine) was then instilled. Instillation was performed using a Rycroft canula inserted on a $1 \mathrm{ml}$ syringe circumferentially under the iris, after injection of sodium hyaluronate (Healon GV) into the anterior chamber to help maintain drug localisation in contact with the ciliary body and iris. Both solutions were prepared and presented in a masked fashion such that neither the surgeon nor the anaesthetist could identify the administered drug The randomisation was devised by an inde- pendent academic pharmacist from the clinical investigation centre, who was not involved in the study. After 10 seconds, the pain rating was immediately repeated when the surgeon restarted the operation. This delay was empirically chosen to allow mepivacaine to exert its anaesthetic effect. If the pain was still considered unbearable after this instillation, a supplementary $1 \mathrm{ml}$ sub-Tenon's injection of $2 \%$ lignocaine or a supplemental sedation with midazolam (0.5-1 mg) were immediately administered. If the pain reappeared before the end of surgery, a second injection of the same solution was instilled by the surgeon. The only medication given at the end of the surgery was gentamicin drops and no patch was applied.

The study was approved by the local ethics committee, Toulouse I.

\section{STATISTICAL ANALYSIS}

Calculation of sample size needed

Referring to the analgesic effect, we assumed that a diminution of at least one point on the scale of the pain chart should be considered as clinically relevant. We anticipated that the placebo effect would produce this result in at least $10 \%$ of the patients and that such an improvement in at least $50 \%$ of the patients receiving the active compound should be considered as a satisfactory result.

Referring to the safety study, the criteria were twofold. On the one hand we knew that the percentage of endothelial cell loss is generally estimated at about $7 \%$ (SD 3\%) after phacoemulsification. ${ }^{13}$ On the other hand we also knew that the inflammatory reaction at day 1 , assessed by the flare values, is generally about 50 (SD 20) photons/ms, in the absence of an intracameral anesthetic treatment. We considered that the treatment would entail an increase of the endothelial cell loss if the percentage of the cellular loss exceeded $10 \%$, and a worsening of the inflammation if the count went up on average to 70 photons $/ \mathrm{ms}$.

According to these hypotheses, and choosing an alpha error of $5 \%$ and a beta error of $90 \%$, we calculated that 50 patients ( 25 in each arm) would be needed to show significant differences in these criteria (one tail test). Fifty consecutive patients were then randomised into two groups, 25 receiving unpreserved $2 \%$ mepivacaine (Carbocaine Astra), 25 receiving the placebo.

\section{Data analysis}

Results are expressed as mean with $95 \%$ confidence interval in parentheses. The level of significance was accepted for $\mathrm{p}<0.05$.

With reference to the efficacy criteria, intragroup analysis comparing the mean pain scores before versus after intracameral injection was performed using the Wilcoxon signed rank test. Intergroup comparison comparing the mean difference in pain scores, before and after the intracameral injection in the placebo versus the mepivacaine group, was performed using the Mann-Whitney $U$ test. The comparison between the two groups of subjects having received an additional local anaesthesia or a 


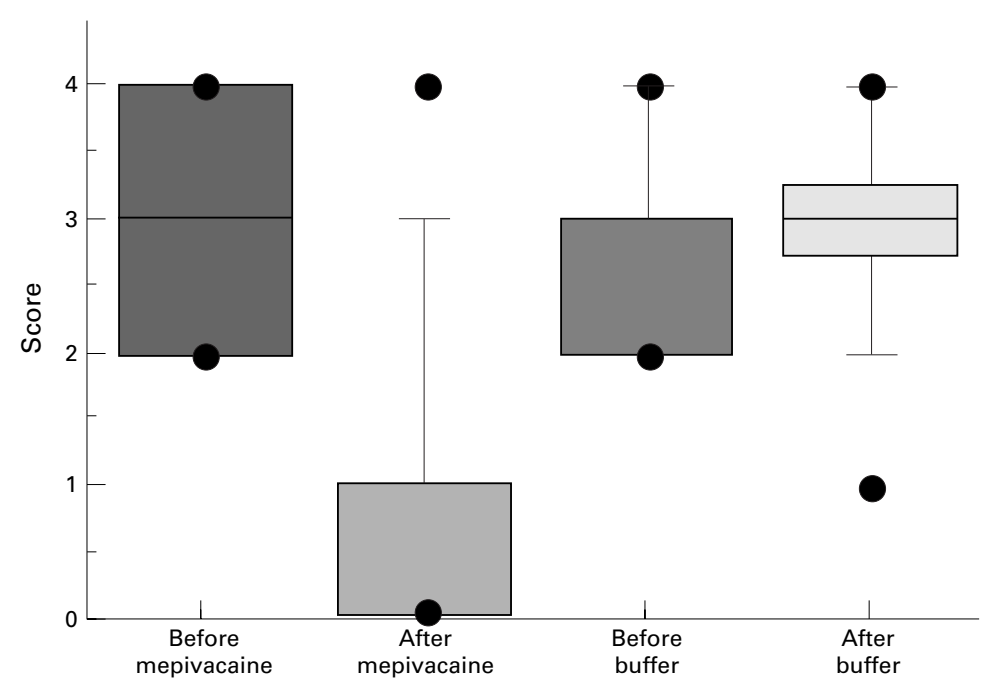

Figure 1 Box plot of pain scores. Shaded areas represent 25th-75th percentile intervals. Lines represent 10th-90th percentile intervals. Black circles represent extreme values.

sedation after the intracameral injection was done with Yates's modified $\chi^{2}$ test.

Comparisons in safety criteria (mean flare values, the IOP values, and the haemodynamic constants between the treated and the control group) were performed using Student's $t$ test. For the comparison of the percentage of cell loss Student's $t$ test was used.

\section{Results}

Fifty patients experienced intraoperative pain and were included in the present study. They were recruited within 12 months out of 227 patients who gave informed consent to participate in the study and underwent preoperative procedure (out of 700 consecutive patients referred to the ophthalmology department of the Toulouse University Hospital for cataract surgery). There was no age and sex difference between the 50 patients who experienced intraoperative pain and those who did not. There was no statistical difference in average mean age between the two treatment groups of patients who actually were randomised to mepivacaine or placebo (mepivacaine, 74 years (range 70-78); placebo, 76 years (74-79)). The sex ratio was similar in both groups (mepivacaine, $15 \mathrm{~F} / 10 \mathrm{M}$, placebo, $14 \mathrm{~F} / 11 \mathrm{M}$ ).

EFFICACY OF INTRACAMERAL MEPICAVAINE

In the 25 patients receiving mepivacaine, 21 $(84 \%)$ noted a decrease of at least one point on the Keele verbal pain chart, while this was observed only in two (8\%) of the control group $\left(\mathrm{p}<10^{-4}\right)$. The average pain score was similar in the two groups before injection of the drug and significantly diminished after intracameral in-

Table 1 Aqueous flare (photon count/ms) following phacoemulsification

\begin{tabular}{lllll}
\hline & \multicolumn{2}{l}{ Flare } & \\
\cline { 2 - 5 } & Preoperative & Day 1 & Day 7 & Day 20 \\
\hline $\begin{array}{l}\text { Mepivacaine } \\
\text { Mean }\end{array}$ & 14.0 & & & \\
$\begin{array}{l}\text { 95\% CI } \\
\text { Placebo }\end{array}$ & $11.0-17.0$ & 28.1 & 19.5 & 13.9 \\
Mean & 12.6 & $20.5-35.7$ & $12.3-26.5$ & $10.6-17.2$ \\
$95 \%$ CI & $10.5-14.7$ & 36.5 & 24.4 & 13.1 \\
\hline
\end{tabular}

jection in the group receiving mepivacaine (mean 3.0 (95\% CI 2.6-3.4) $0.8(0.3-1.3)$ $\mathrm{p}<10^{-4}$ ) while remaining unchanged in the control group $(2.9(2.6-3.2) v 2.9(2.5-3.3))$

(Fig 1). The mean reduction in pain score was statistically significant between the two groups $\left(2.1(1.6-2.6)\right.$ v $0.0(-0.3$ to +0.3$\left.) \mathrm{p}<10^{-4}\right)$. Sixteen patients needed additional medication in spite of intracameral injection - two in the mepivacaine group and 14 in the control group $\left(\mathrm{p}<10^{-3}\right)$.

Pain relief occurred extremely rapidly in the mepivacaine group, within the first 10 seconds in all responders. Pain relief was sustained during the following course of surgery in 19 of the 21 responders. However, in two patients, a second intracameral injection was performed, because pain reoccurred before the end of the surgery. This second injection allowed the surgery to be completed.

TOLERANCE OF INTRACAMERAL MEPIVACAINE

Average preoperative endothelial cell counts were similar in both groups (mepivacaine 2297 (range 2163-2431); placebo 2274 (21322416)). There was a mean 5 (95\% CI 1-9) reduction of endothelial cell counts in the mepivacaine group, compared with a mean 7 (3-11) reduction in the placebo group, the difference not being significant.

Patients developed no or a moderate postoperative inflammatory reaction (0 or $1+$ for clinical flare or cells). The flare values using the laser flare meter are presented in Table 1. They were significantly higher on the first postoperative day, declining at day 7 with a recovery to preoperative levels at day 20. At each visit, there was no statistical difference between the two groups.

Similarly, there was no significant difference in IOP between the two groups. The values of the IOPs in the mepivacaine and control groups were respectively $14.9(13.8-16.0)$ and 14.8 (13.9-15.7) preoperatively, 14.9 (14.0$15.8)$ and $14.9(13.5-16.3)$ at day $1,15.2$ (14.2-16.2) and $14.1(13.1-15.1)$ at day 7 , and $14.1(13.4-14.8)$ and $13.6(12.7-14.5)$ at day 30.

Heart rate, diastolic and systolic blood pressure did not significantly differ between the placebo and mepivacaine groups at any time before, during and after phacoemulsification (data not shown).

\section{Discussion}

This prospective placebo controlled randomised double blinded study documents the significant complementary analgesic efficacy of an intracameral intraoperative administration of $2 \%$ unpreserved mepivacaine in patients undergoing phacoemulsification under topical anaesthesia. Indeed, most patients had a rapid reduction of pain intensity to a tolerable level within less than 10 seconds. A majority (13 out of 25) experienced a complete disappearance of the pain. Four patients however were not improved by mepivacaine. Their general and ophthalmological conditions did not differ from those of the others and we have no satisfactory explanation as to why mepivacaine did 
not exert its desired effect in these patients. In most patients, the anaesthetic effect of intracameral injection of mepivacaine lasted long enough to allow normal termination of surgery. However, in two patients, pain reappeared before the end of surgery, within approximately 10-15 minutes. In these cases, a second intracameral injection proved to be as effective as the previous one, without tolerance.

Furthermore, our procedure proved to be safe, as we did not observe any significant adverse events. The average amount of endothelial cell loss was similar in the mepivacaine and placebo groups, being approximatly $6 \%$, a percentage corresponding to that reported in other studies in a similar surgery. ${ }^{13}$ Similarly, our results also show the absence of induction of any inflammatory reaction or elevation of IOP, after the operation and in the longer term follow up. Additionally, although a systemic distribution of the drug was likely, we did not detect any significant change in heart rate, systolic, and diastolic blood pressure compared with placebo, probably because of the small dose injected intracamerally.

Gills and colleagues ${ }^{4}$ recently reported for the first time the usefulness of an intracameral injection of an anesthetic, lignocaine, during cataract surgery under topical anaesthesia. These authors documented the analgesic efficacy of this procedure versus placebo in 301 patients. These results were then reproduced by several authors in recent studies..$^{5-7}$ As in the present study, the authors did not observe any significant adverse event. The results of the present trial concentrate on two main differences compared with these previously published studies.

The first difference is that Gills and colleagues, ${ }^{4}$ as well as Koch, ${ }^{5}$ Tseng and Chen, ${ }^{6}$ and Crandall et $a l,{ }^{7}$ performed a systematic a priori intracameral injection in every patient undergoing phacoemulsification under topical anaesthesia. But, we only injected those who complained of pain during the course of surgery, representing only $5-10 \%$ of patients. Our study demonstrates the rapid onset of the analgesic effect of mepivacaine when it is administered facing the ciliary body. This rapidity of action is compatible with a curative rather than a preventative philosophy towards pain in such conditions. Therefore, we feel that it is not necessary to perform an intracameral injection systematically in all patients, and that it may be reasonable and cost effective to inject mepivacaine only if and when a patient complains of pain.

The second difference is that we used a different anaesthetic drug. When deciding to deliver an anaesthetic drug intracamerally, we prefered mepicavaine to lignocaine because, although mepicavaine is reported to have a similar time of onset and degree of sensory blockade $^{914}$ it may have a longer duration of action $^{8-10}$ and somewhat fewer toxic properties. ${ }^{10}{ }^{14}$ Both drugs are effective when injected intracamerally. Comparative pharmacological studies, particularly assessing their respective onset and duration of action after injection into the anterior chamber, would be welcome.

The present results suggest that additional intraoperative intracameral mepivacaine is of assistance in the case of cataract surgery performed under topical anaesthesia. It is probably unnecessary to give such complementary anaesthesia systematically at the beginning of surgery in every patient, and it may be reasonable to reserve this procedure only for those who complain of pain during the course of the surgery. An intraoperative intracameral mepivacaine injection might also be helpful during painful phacoemulsification performed under locoregional anaesthesia. Its strong and immediate analgesic effect should allow the successful completion of the phacoemulsification in difficult cases. In fact, after the positive results of this study became available, we used intracameral mepivacaine in several patients suffering pain during phacoemulsification under locoregional anaesthesia, with a remarkable efficacy in all of them. Further controlled studies would be necessary to document this possible additional indication.

Presented at the ASCRS, ASOA Boston April 1997.

The authors have no proprietary interest in any research or materials presented in this article.

1 Kershner RM. Topical anaesthesia for small incision self-sealing cataract surgery. A prospective evaluation of the first 100 patients. F Cataract Refract Surg 1993;19:290-2.

2 Grabow HB. Topical anaesthesia for cataract surgery. Eur 7 Implant Refract Surg 1993;5:20-34.

3 Williamson CH. Topical anaesthesia using lidocaine. In: Fine IH, Fichman RA, Grabow HB, eds. Clear-corneal cataract surgery and topical anaesthesia. Revised ed. Thorofare, NJ: Slack, 1993:121-8.

4 Gills JP, Cherchio M, Raanan MG. Unpreserved lidocaine to control discomfort during cataract surgery using topical anaesthesia. I Cataract Refract Surg 1997;23:545-50.

5 Koch PS. Anterior chamber irrigation with unpreserved lidocaine $1 \%$ for anaesthesia during cataract surgery. $\mathcal{f}$ Cataract Refract Surg 1997;23:551-4.

6 Tseng SH, Chen FK. A randomized clinical trial of combined topical-intracameral anesthesia in cataract surgery. Ophthalmology 1998;105:2007-11.

7 Crandall AS, Zabriskie NA, Patel BC, et al. A comparison of patient comfort during cataract surgery with topical anesthesia versus topical anesthesia and intracameral lidoanesthesia versus topical anesthesia and

8 Schimek F, Steuhl KP, Fahle M. Retrobulbar blockade of somatic, motor, and visual nerves by local anesthetics. Ophthalmic Surg 1993;24:171-80.

9 Bromage PR, Gertel M. An evaluation of two new local anaesthetics for major conduction blockade. Can Anaesth Soc F 1970;1 17:557-64.

10 Ulfendahl HR. Some pharmacological and toxicological properties of a new local anaesthetic, carbocaine. Acta Anaesthesiol Scand 1957;1:81-6.

11 Keele KD. The pain chart. Lancet 1948;iii:6-8.

12 Hogan MJ, Kimura SJ, Thygeson P. Signs and symptoms of uveitis. Am f Ophthalmol 1959;47:155-70.

13 Abbott RL, Forster RK. Clinical specular microscopy and intraocular surgery. Arch Ophthalmol 1979;97:1476-9.

14 Henn F. Determination of the toxicological and pharmacological properties of carbocaine, lidocaine and procaine by means of simultaneous experiments. Acta Anaesthesiol Scand 1960;4:125-54. 